\title{
Ungarische Kläger in Wien \\ Die Tätigkeit der Ungarischen Hofkanzlei auf dem Gebiet der Gerichtsbarkeit (1526-1727)
}

\author{
Hungarian Plaintiffs in Vienna. The Hungarian Court Chancery's Activities in Judicial Affairs \\ (1526-1727)
}

Although as early as 1464 Matthias Corvinus had separated the part of the Hungarian Chancery that dealt with judicial affairs (cancellaria minor, tabula regia) from the rest, the Hungarian Court Chancery (cancellaria maior, Ungarische Hofkanzlei) in the Habsburg era still exercised some competences in this field. In addition to the issuing of royal pardons and the participation in courts of arbitration and extraordinary tribunals, a vast array of royal mandates enabled the Chancery to interfere in lawsuits in almost any state of the proceedings. Based on archival sources mainly from Budapest and Vienna, the article offers an overview of the development of the institution and its considerable judicial activities from 1526 until its reform in 1727, which more or less put an end to its involvement in jurisdiction, even if not completely. In addition, the development of the Chancery's personnel - offices, numbers, career patterns - is analysed.

Keywords: Hungarian Court Chancery - jurisdiction - mandate - pardon - Hungary - Habsburg era

\section{Einführung}

Das Königreich Ungarn war von 1526 bis 1918 Teil der Habsburgermonarchie. Trotz aller Zentralisierungsbestrebungen der Herrscherfamilie und der Zentralverwaltung konnten die Länder der Stephanskrone in vielen Gebieten ihre Selbständigkeit bewahren. Dazu gehörte das Justizwesen, was die Fortgeltung der besonderen ungarischen Rechtsgewohnheiten erleichterte. ${ }^{1}$ Es waren nur einige spezielle Bereiche des Justizwesens bzw. bestimmte historische Konstellationen, in denen die österreichischen Erbländer bzw. die Zentralverwaltung das ungarische Rechtswesen beeinflussen konnten. Der Fall des österreichischen Strafgesetzbuches (sanktioniert

${ }^{1}$ Zur Entwicklung des ungarischen Justizwesens TIMON, Ungarische Verfassungs- und Rechtsgeschichte; RADVÁNSZKY, Grundzüge.
1656), das großen Einfluss auf die ungarische Strafgerichtspraxis ausübte, ist daher eher als Ausnahme anzusehen: Ab 1696 erschien es als ständiger Anhang des ungarischen Corpus Juris. ${ }^{2}$ Von den Zentralorganen waren es vor allem der Hofkriegsrat (Militärgerichtsbarkeit) und die Hofkammer, die gewisse Kompetenzen im Gebiet der Rechtsprechung besaßen. Letztere war für Schmuggel - zeitgenössisch formuliert: Kontraband-Angelegenheiten - zuständig. ${ }^{3}$ Die stürmischen Jahrzehnte der Regierungszeit Leo-

\footnotetext{
${ }^{2}$ KovÁCs, Zur Geschichte des ungarischen Strafrechts 23; MAYER, Verwaltungsreform in Ungarn 108.

${ }^{3}$ Siehe z.B. die Untersuchungsakten in der Angelegenheit der Oberpullendorfer Adeligen und Leibeigenen, die Vieh aus Ungarn nach Niederösterreich schmuggelten (1576), ÖStA, AVA FHKA, HKA, HFU, r. Nr. 31, Konv. 1576 Apr., fol. 42-46, r. Nr. 32, Konv. 1576 Aug., fol. 117-126, 205-206, 424-435.
} 
polds I. (1657-1705) brachten auch einige Eingriffe in die Gerichtsbarkeit des Königreichs Ungarn. Hier ist vor allem die Tätigkeit der Neoacquistica Commissio (gegründet 1689) zu erwähnen, die über die Ansprüche der früheren Besitzer der zurückeroberten ungarischen Gebiete entschied. ${ }^{4}$ In den Jahren der Rückeroberungskriege (1683-1699) konnten der Hofkriegsrat und die Hofkammer ihren Einfluss ausdehnen und lösten damit Beschwerden der ungarischen Stände aus. ${ }^{5}$ Die zurückeroberten Gebiete wurden in den letzten Jahrzehnten des 17. Jahrhunderts eigenen Verwaltungsorganen, den sog. Kameraladministrationen, unterstellt und von ihnen verwaltet. Diese Administrationen besaBen provisorisch das Recht, die Gerichtsbarkeit in Zivilsachen auszuüben. ${ }^{6}$ Ein spezieller Fall war das Obersthofmarschallamt. 1708 beschwerten sich die ungarischen Stände beim Kaiser, dass das Obersthofmarschallamt nicht nur in Nachlassverfahren der Mitglieder der Ungarischen Hofkanzlei, sondern auch anderer Ungarn, die in Wien gestorben waren, tätig wurde. ${ }^{7}$ Schließlich ist auf die Tätigkeit der außerordentlichen Gerichte (judicia delegata) hinzuweisen, z.B. den unter dem Vorsitz des österreichischen Hofkanzlers Johann Paul Hocher stehenden Gerichtshof, der in der Angelegenheit der ungarischen und kroatischen Hochadeligen Ferenc (Franjo) Frangepán, Péter (Petar) Zrínyi und Ferenc Nádasdy, die in die Magnatenverschwörung involviert waren, eingesetzt wurde $(1670-1671){ }^{8}$

\footnotetext{
${ }^{4}$ MEzŐsI, A fegyverjog (jus armorum) megváltása 179-193; BÓNIS, DEGRÉ, VARGA, A magyar bíróság szervezet 50; Soós, Pest-Pilis-Solt vármegye és a Neoacquistica Commissio 17-51.

${ }^{5}$ BÓNIS, A bíróság szervezet megújítása 40.

${ }^{6}$ MAYER, Verwaltungsreform in Ungarn 17-19.

${ }^{7}$ BÓNIS, A bíróság szervezet megújítása 40.

${ }^{8}$ ReDLICH, Weltmacht des Barock 209-211.
}

\section{Der Personal und die cancellaria minor}

In der Frühen Neuzeit blieben die Ämter aller vier Großrichter des Landes (judices regni ordinarii), deren Aufgabenbereiche sich schon im Spätmittelalter herauskristallisiert hatten, erhalten. ${ }^{9}$ Der Palatin bzw. Statthalter war für Angelegenheiten der Magnaten zuständig, weiters in Fällen mit politischem Charakter; er führte den Vorsitz in der Septemviraltafel, die als oberstes Appellationsgericht fungierte. Der Judex Curiae oder Oberste Landesrichter behandelte Erbangelegenheiten ausgestorbener Familien (sog. defectus seminis) und saß dem Appellationsgericht für kroatisch-slavonische Angelegenheiten vor; er sollte in Oberungarn residieren und als Richter in diesem entlegenen Landesteil, der schwer von den Zentren Wien und Pressburg [Bratislava] aus zu regieren war, tätig sein. Der dritte Großrichter war der Tavernicus (Oberschatzmeister, magister tavernicorum), der als Leiter des Appellationsforums für einige königliche Städte fungierte. Für das Thema des vorliegenden Beitrages von besonderem Interesse ist jedoch der vierte Großrichter, der Personal (personalis praesentiae regiae in judiciis locumtenens), der seit dem 15. Jahrhundert in Vertretung des Königs Recht sprach. Matthias Corvinus trennte 1464 die Abteilung der Hofkanzlei, die sich mit der Gerichtsbarkeit beschäftigte, von den anderen Kanzleiabteilungen ab und verselbständigte sie unter der Leitung des Personals. ${ }^{10}$ Diese Institu-

\footnotetext{
${ }^{9}$ Einen guten Überblick über die ungarische Gerichtsorganisation bzw. Rechtspraxis vermittelt eine $\mathrm{Ab}$ handlung, die um 1670 von einem Unbekannten zusammengestellt wurde: "Memoriale circa judices Regni Processum juris in Tabula Regia sedibus spiritualibus et comitatibus, ac alijs judicibus Regni Ungariae. Ac quis modus procedendi defacto oberservari possit?", ÖStA, HHStA, Handschriftensammlung W 185 (=Böhm 312), fol. $428-439$, bes. fol. $428^{\mathrm{r}}-429^{\mathrm{r}}$.

${ }^{10}$ KuBINYI, Matthias Rex 49-50; BÓNIS, DEGRÉ, VARGA, A magyar bíróság szervezet 18 .
} 
tion wurde im 16. Jahrhundert noch oft als cancellaria minor, später als tabula regia (königliche Tafel) bezeichnet. Es handelt sich um ein Gericht mit einer sehr umfassenden Zuständigkeit, das sowohl erstinstanzlich als auch als Appellationsgericht tätig war. Die Bedeutung dieser Institution kommt schon allein darin zum Ausdruck, dass der Personal gleichzeitig zwei Protonotare (d.h. Richter) anstellen konnte und musste, die anderen Großrichter dagegen jeweils nur einen. Der Personal saß nicht nur der Königlichen Tafel vor, sondern führte auch den Vorsitz in der unteren Tafel der ungarischen Ständeversammlung (Landtag/Reichstag). Über seine Tätigkeit wissen wir wenig. Er residierte in Ungarn, meist auf einem seiner in Westungarn liegenden Güter. Er hatte das Recht, im Namen des Königs Urkunden ausstellen zu lassen, und führte das richterliche Siegel des Königs, das ihm anvertraut war. ${ }^{11}$

\section{Die Ungarische Hofkanzlei (1527-1727)}

Die Lage der Ungarischen Hofkanzlei, die im Zeitalter der Jagiellonen das Zentralorgan des Königreichs gewesen war, veränderte sich mit der Thronbesteigung von Ferdinand I. (15261564) grundsätzlich. ${ }^{12}$ Die Eingliederung der ungarischen Verwaltung in das neue Staatsgebilde war an sich schon eine ernste Herausforderung, die durch die Doppelkönigwahl und den folgenden Bürgerkrieg mit seinen unsicheren inneren Verhältnissen noch enorm verschärft wurde. Zu einer grundlegenden Ände-

\footnotetext{
${ }^{11}$ Leider hat die Tätigkeit des Personals bisher wenig Aufmerksamkeit in der Forschung gefunden, so dass sie als vollständig unbearbeitet gelten kann.

${ }^{12}$ Die folgende Darstellung basiert auf eigenen Forschungen, die vor dem Abschluss stehen. Darin fokussiere ich die Geschichte der Ungarischen Hofkanzlei von 1526 bis 1690. FAZEKAS, Geschichte 49-65, DERS., Humanisten und Juristen 321-331.
}

rung der Verhältnisse kam es erst in den 1540er Jahren, als sich Ferdinand I. nach der osmanischen Eroberung von Buda (1541) der Neuorganisation der ungarischen Verwaltung widmete. Im Rahmen dieser Bemühungen wurde auch die Ungarische Hofkanzlei neu formiert. Durchgeführt wurde diese Reform von Nikolaus Oláh (1497-1568), dem damaligen Vizekanzler und späteren Erzbischof von Gran (1553-1568) sowie Statthalter (1562-1568), der zuvor als Sekretär von Königin Maria tätig gewesen war und in Ungarn und in den Niederlanden vielfältige bürokratische Erfahrung gesammelt hatte. Oláh organisierte die Hofkanzlei nach dem Beispiel der Hofkanzlei der Jagiellonen vor 1526. Diese Organisation der Ungarischen Hofkanzlei blieb bis 1690 im Wesentlichen unverändert.

Die Kanzlei verfügte in dieser Zeit über wenig Personal. Ihre Leitung oblag einem Hofkanzler, der bis 1732 mit wenigen Ausnahmen ein Kleriker war. Im 16. Jahrhundert waren darüber hinaus Vizekanzler tätig, insbesondere dann, wenn kein Kanzler ernannt worden war; im folgenden Jahrhundert hingegen gab es nur "ad hoc" Vizekanzler (cancellarius substitutus) im Fall einer Verhinderung des Kanzlers. Der wichtigste Amtsträger der Hofkanzlei war der Sekretär (im 16. Jahrhundert waren oft zwei Sekretäre gleichzeitig tätig). Er organisierte und leitete den Geschäftsgang der Kanzlei, bereitete die einzelnen Angelegenheiten zur Entscheidung vor und konzipierte die Erledigungen gemäß der kaiserlichen Entscheidung (Konzipisten wurden erst nach 1690 angestellt). Der dritte Funktionsträger der Hofkanzlei war der Registrator, der gleichzeitig die Funktion des Siegelbewahrers (conservator sigilli) innehatte. Der Registrator führte die Königlichen Bücher (Libri regii). Seit dem 17. Jahrhundert war er auch für juristische Angelegenheiten zuständig, wodurch der mit Aufgaben überhäufte Sekretär entlastet werden konnte. Neben dem Kanzler, dem Sekretär und dem Registrator waren in der Hofkanzlei noch zwischen drei und sieben Schreiber (jurati notarii 
oder scribae) tätig. Diese Schreiber waren Privatangestellte (!) des jeweiligen Kanzlers, der Sekretäre oder des Registrators, so dass ihre Amtszeit mit dem Tod der sie beauftragenden Amtsträger endete. Von den genannten Amtsträgern der Kanzlei bezog nur der Sekretär ein staatliches Gehalt. Der Kanzler lebte vor allem von seinen kirchlichen Pfründen, außerdem von Taxeinnahmen; die anderen Mitglieder der Kanzlei erhielten ausschließlich Zahlungen aus Taxeinnahmen.

Diese stark mittelalterliche Organisation war den Herausforderungen der Neuzeit immer weniger gewachsen. In der Zeit der Rückeroberungskriege (1683-1699) wurde deutlich, dass man die Reform der Hofkanzlei nicht weiter aufschieben konnte. Sie erfolgte 1690; die Kanzlei bekam eine Instruktion (erstmals in ihrer Geschichte), wurde zur Kollegialbehörde umgestaltet (außer dem Vizekanzler wurden auch vier Räte ernannt), das Personal wurde vermehrt und mit einem staatlichen Gehalt versehen. 1727 waren schon 26 Personen angestellt.

$\mathrm{Zu}$ den Aufgaben der Ungarischen Kanzlei gehörten nach wie vor die Ausübung der königlichen Majestätsrechte und der Schutz der königlichen und staatlichen Interessen. Dementsprechend stellte sie die Urkunden über königliche Donationen aus und bestätigte die Donationsbriefe der Würdenträger des Landes. Im Prinzip verkehrte der Monarch mit den ungarischen Regierungsorganen bzw. mit den ungarischen ständischen Würdenträgern über die Ungarische Hofkanzlei. Auf dem Gebiet der auswärtigen Angelegenheiten verfügte die Kanzlei lediglich über geringen Einfluss. So erfolgte beispielsweise die Präsentation (Nomination) der ungarischen Bischöfe an die päpstliche Kurie durch die Ungarische Hofkanzlei. In Angelegenheiten ungarischer Untertanen trat der habsburgische Herrscher auch dem polnischen König über Ausfertigungen der Ungarischen Hofkanzlei gegenüber. Als glaubwürdige Stelle durfte die Kanzlei auch gewisse notarielle Funktionen aus- üben. Sie war berechtigt, Privaturkunden, Testamente usw. zu bekräftigen.

Die Bedeutung der Ungarischen Hofkanzlei bestand darin, dass sie das einzige ungarische Verwaltungsorgan war, das in unmittelbarer und regelmäßiger Verbindung mit dem Herrscher stand. Deshalb residierte die Hofkanzlei in Wien - in der Rudolfinischen Ära, aufgeteilt in zwei Abteilungen, in Wien und Prag - bzw. begleitete den Herrscher auch auf seinen Reisen. ${ }^{13}$ Einen ständigen Sitz bekam die Ungarische Hofkanzlei erst $1747 .{ }^{14}$ Diese Tatsache wirkte sich sehr negativ auf die Erhaltung des Aktenmaterials aus und erschwert heute die Erforschung der Geschichte der Institution.

\section{Die Tätigkeit der Ungarischen Hofkanzlei auf dem Gebiet der Gerichtsbarkeit}

Trotz der Abspaltung der cancellaria minor von der Hofkanzlei, auch cancellaria maior genannt, verblieben der Hofkanzlei einige Aufgaben im Justizwesen.

I. Der König verfügte über das Recht der Begnadigung. Die im Archiv der Ungarischen Hofkanzlei aufbewahrten Dokumente beweisen, dass er dieses Recht regelmäßig durch die Ungarische Hofkanzlei ausübte. Die Verurteilten wandten sich mit Supplikationen an den König, die von der Hofkanzlei bearbeitet, zusammengefasst, mit einer Stellungnahme versehen und in Audienz vorgetragen wurden. Die Entscheidung des Herrschers wurde in eine von der Ungarischen Hofkanzlei ausgestellte Urkunde gefasst. Aus den Jahren 1638 und 1639 haben sich die sog. Audienzzettel erhalten. Unter den damals vorgetragenen Angelegenheiten finden

\footnotetext{
${ }^{13}$ FAZEKAS, Geschichte 51.

${ }^{14}$ MrAZ, Palais 54-55.
} 
sich auch Gnadengesuche. ${ }^{15}$ Inhaltlich sind diese Fälle sehr verschieden: meistens ging es um eine (angeblich) fahrlässige Tötung, Hexerei, Inzest, Kirchendiebstahl etc.

II. In einzelnen Fällen konnte die Hofkanzlei bzw. konnten ihre Amtsträger als Mitglied eines Schiedsgerichtes tätig werden. In mindestens zwei Fällen nahm sie dieses Recht in Anspruch. 1670 lud der Hofkanzler auf Antrag von Georg Horváth die Stadt Győr [Raab] vor, um in einer Streitfrage eine freundschaftliche Vereinbarung zwischen den Parteien zu erreichen. ${ }^{16} 1675$ kam vor dem Hofkanzler Tamás Pálffy, dem Bischof von Neutra [Nyitra] und Matthias Hedly in Wien eine Vereinbarung zwischen den Parteien in der Auseinandersetzung um das Testament von Gräfin Elisabeth Batthyány (verheirate Erdődy) zustande. ${ }^{17}$

III. Die Ungarische Hofkanzlei wirkte außerdem an den judicia delegata, also vom Herrscher delegierten Gerichten mit, die in speziellen Fällen urteilten. So trat der ungarische Hofkanzler z.B. in der Zeit Rudolfs II. als Richter in den Prozessen auf, die gegen ungarische Magnaten geführt wurden und die am Ende in den BocskayAufstand (1605) mündeten. Der Hofkanzler Márton Pethe de Hetes, Bischof von Győr, wurde 1601 in einem Prozess gegen György Drugeth de Homonna und seine Mutter zum Richter ernannt. In diesem Fall handelte es sich um eine Art Notlösung, da sich der Landesrichter István

\footnotetext{
${ }^{15}$ MNL OL, MKL A 33 Propositiones et opiniones 1638: 1 (Mord), 31 (Mord), 89 (Ehebruch), 93 (Mord), 197 (Mord); 1639: 32 (Diebstahl in einer protestantischen Kirche), 74 (Mord), 78 (Mord), 171 (Hexerei), 262 (Mord), 268 (Inzest). Konzept eines Gnadenbriefes für den Mörder Michael Sztrecsényi, 21. 8. 1679, Wien. MNL OL MKL A 35 Conc. exp. 1679: 164.

${ }^{16}$ Hofkanzler Tamás Pálffy, Bischof von Neutra, an die Stadt Győr, 1. 11. 1670, Wien. Orig. ungarisch. Győr Megyei Jogú Város Levéltára [Archiv der Stadt Győr] IV.A. 1001/H Levelezések Kart. 1.

${ }^{17}$ MNL OL MKL A 35 Conc. exp. 1675: 302.
}

Báthori und der Propalatin Miklós Istvánffy, die in Fällen von Hochadeligen zuständig gewesen wären, anscheinend nicht auf einen Prozess einlassen wollten. Der Hofkanzler dagegen hatte dabei offensichtlich keine Skrupel. ${ }^{18}$

IV. Die Mandate. Die ungarischen Rechtshistoriker sind einhellig der Meinung, dass die Gerichtsbarkeit in Ungarn am Ende des 17. Jahrhunderts als einigermaßen chaotisch $\mathrm{zu}$ bezeichnen ist. Gerichtssessionen, die sog. octavalia, fielen häufig aus (besonders nach 1650), die Protonotare der einzelnen Großrichter übten die Rechtsprechung nach Belieben aus und sprachen oft fragwürdige Urteile. Ein weiteres Übel des damaligen ungarischen Justizwesens war, dass der Prozessverlauf durch Mandate der Großrichter bzw. des Königs zu beeinflussen war (sog. mandata iudiciaria). ${ }^{19}$ Diese Mandate sind ein Charakteristikum des ungarischen feudalen Rechtssystems. Der Würdenträger, der berechtigt war das Mandat zu erlassen, wies damit die untergeordneten Justizorgane an, einen bestimmten Rechtsakt (eine Rechtshandlung) zu verrichten. Das Mandat fungierte als Rechtsmittel; es sollte helfen, Fehler der unteren Gerichte zu korrigieren. Es existierten viele verschiedene Arten von Mandaten; nach Auffassung eines Rechtshistorikers existierten ebenso viele Arten von Mandaten wie Angelegenheiten. Jedes Mandat musste gewisse formalisierte Bestandteile enthalten, auf die ich hier nicht eingehen möchte. ${ }^{20}$ Entscheidend ist im vorliegenden Zusammenhang, dass die königlichen Mandate von der Ungarischen Hofkanzlei ausgestellt wurden.

\footnotetext{
${ }^{18}$ Bericht der Ungarischen Kammer an Kaiser Rudolf II., 22. 9. 1601, Pressburg. ÖStA, AVA FHKA, HKA, HFU r. Nr. 70, Konv. 1570 Aug., fol. 243-246. 19 TORDAY, A megyei polgári peres eljárás 136-142; BÓNIS, DEGRÉ, VARGA, A magyar bírósági szervezet 112

${ }^{20}$ TORDAY, A megyei polgári peres eljárás 139-141.
} 
Die Durchsicht der erhaltenen Konzepte aus den Jahren zwischen 1670 und 1690 brachte eine Reihe von verschiedenen Mandaten zum Vorschein:21

- mandatum admonitorium (Ermahnung des Beklagten durch den Richter noch vor Prozessbeginn)

- mandatum cassatorium (Kassation eines Urteils)

- mandatum compulsorium (Befehl, Zeugen zu verhören)

- mandatum dilatorium/prorogatorium (Prozessaufschub)

- mandatum evocatorium (Vorladung)

- mandatum executorium (Befehl zur Vollstreckung eines Urteils)

- mandatum inhibitorium (Anordnung, einen Prozess nicht weiterzuführen, z.B. wegen Unzuständigkeit des Richters oder Formfehlern etc.)

- mandatum praeceptorium (Anordnung der Aufnahme eines Prozesses)

- mandatum novum cum gratia (Anordnung der Wiederaufnahme eines Prozesses)

- mandatum relaxatorium (Aufhebung des mandatum inhibitorium)

Wie leicht $\mathrm{zu}$ sehen ist, ließ sich durch diese Befehle in alle Prozessphasen eingreifen. Schon vor Beginn des Prozesses konnte eine Mahnung ergehen (mandatum admonitorium). Ein Prozess ließ sich durch Mandate aufnehmen (mandatum praeceptorium, evocatorium) und zum Stillstand bringen (mandatum inhibitorium), dieser Stillstand konnte wieder aufgehoben (mandatum relaxatorium) oder ein Prozessaufschub erwirkt werden (mandatum dilatorium/prorogatorium). Darüber hinaus war es möglich, einen Richter zur Vollstreckung eines Urteils anzuweisen (mandatum executorium), aber ebenso, das Urteil zu kassieren (mandatum cassatorium) und das

${ }^{21}$ MNL OL MKL A 35 Conc. exp. Kart. 17-27. ganze Verfahren wieder aufnehmen zu lassen (mandatum novum cum gratia, revisio causae).

Die Frage, wie hoch der Anteil der gerichtlichen Agenden an der gesamten Tätigkeit der Ungarischen Hofkanzlei zu veranschlagen ist, ist nicht leicht $\mathrm{zu}$ beantworten, weil das Archiv der Kanzlei vor 1690 stark skartiert wurde. Aufgrund der erhaltenen Konzepte aus den Jahren 1670-1690 schätze ich diesen Anteil auf zwischen 25 und 40 Prozent. Diese Schätzung wird durch den Protokollband des Zentralbestandes der Hofkanzlei (Conceptus expeditionum) für das Jahr 1696 bestätigt. Danach entstanden in diesem Jahr 932 Konzepte. Etwa 250 Stücke lassen sich unter dem Schlagwort Gerichtsbarkeit einordnen, was einem Anteil von 27 Prozent entspricht. $^{22}$

Die Bearbeitung aller dieser Justizsachen bedeutete sicherlich eine Belastung für das wenig zahlreiche Personal der Kanzlei. Auf der anderen Seite ist aber in Rechnung $\mathrm{zu}$ stellen, dass die auszustellenden Stücke in Gerichtssachen stark formalisiert waren und daher von einem Kanzleiangestellten mit Hilfe eines Formelbuches relativ schnell verfertigt werden konnten. Im Formularium von Benedikt Zercheky, das in den 1560er Jahren zusammengestellt wurde, finden sich etwa 30 Schriftstücke (von insgesamt 350), die etwas mit Gerichtsbarkeit zu tun haben. ${ }^{23}$ Manchmal blieb dem königlichen Sekretär auch kaum etwas zu tun übrig, weil der Kläger bzw. in diesem Fall der Beauftragte der Klägerin seinem Antrag das Konzept des gewünschten Mandates bereits beigefügt hatte, so etwa János Thamásfalvi in seinem Antrag an den Sekretär János Maholányi 1689.24 Trotz der

22 MNL OL MKL A 34 Conc. exp. Bd. 474.

${ }^{23}$ KOVACHICH, Formulae solemnes styli LVIII-LXXXV. ${ }^{24}$ Thamásfalvi war im Auftrag der Witwe von Dr. Khien tätig, die gegen Miklós und János Pálffy prozessierte. Schreiben von Thamásfalvi an János Maholányi, 5. 6. 1689, Pressburg. Orig. ungarisch. MNL OL MKL A 97 Hungarica et Transylvanica 
damit verbundenen Arbeitsbelastung hatte das Personal der Hofkanzlei wahrscheinlich nicht viel gegen die Ausstellung der Mandate einzuwenden, weil die Antragsteller meist bereit waren, sich für diese Bemühungen zu revanchieren. Der junge Pál Esterházy etwa, der in den 1650er Jahren nach dem Tod seines älteren Bruders László einen langen Kampf um die Erhaltung der Familiengüter führte, brauchte schnell ein mandatum inhibitorium - und versprach dem Sekretär der Hofkanzlei János Ruttkay dafür eine würdige Gegenleistung. ${ }^{25}$

\section{Personelle Verflechtungen zwischen der Ungarischen Hofkanzlei und den ungarischen Obergerichten}

Die Tätigkeit der Ungarischen Hofkanzlei auf dem Gebiet Rechtsprechung brachte mit sich, dass von ihren Mitarbeitern auch juristische Kenntnisse verlangt wurden. Diese Qualifikationsanforderung stellte eine Verbindung zwischen dem Personal der Kanzlei und dem der Obergerichte her. Es gibt sowohl Kanzleiangestellte, die ihre Karriere im Dienst eines Personals oder eines Protonotars begonnen hatten, als auch solche, die ihre Laufbahn an einem Obergericht fortsetzten. Michael Szikszay, der spätere Registrator der Ungarischen Hofkanzlei, war zunächst als Schreiber im Dienst des Personals Johann Joó tätig (1587-1603), wurde später Kanzleischreiber und von 1615 bis 1636 Registrator und gleichzeitig Siegelbewahrer. ${ }^{26}$ In

Kart. 9, Schriften Leopold I. Iuridica: Causae iudiciales, fol. 196-197.

${ }^{25}$ Pál Esterházy an den königlichen Sekretär András Ruttkay, 17. 4. 1657. Orig. ungarisch. MNL OL MKL A 97 Hungarica et Transilvanica Kart. 9, Schriften Leopold I. Iuridica: Causae iudiciales, fol. 214-215.

${ }^{26}$ Königliche Bestätigung über den Besitz eines Hauses im Markt Tarcal, 18. 4. 1603, Prag. MNL OL MKL A 57 Libri regii vol. 5, pag. 818-819. der Zeit von Szikszay vergrößerte sich die Bedeutung des Amts des Registrators, oft auch vicesecretarius genannt. Die erhaltenen Konzepte aus dem Geschäftsgang zeigen, dass der Registrator nun auch die Erledigung der einfacheren Angelegenheiten, vor allem jener mit juristischem Charakter, übernommen hatte. Diese Kompetenzerweiterung war notwendig, weil nach 1610 nur noch ein einziger Sekretär in der Ungarischen Hofkanzlei angestellt war, der in irgendeiner Form entlastet werden musste. Es ist also kein Zufall, dass drei der nächsten vier Registratoren vor ihrer Anstellung in der Hofkanzlei niedere Beamte bei einem Obergericht gewesen waren. Pál Lászlóffy war Notar (Schreiber) der Königlichen Tafel (1635). ${ }^{27}$ Miklós Dvornikovich stand als Schreiber im Dienst des Adam Cziráky, Protonotar des Palatins Pál Pálffy (1653). ${ }^{28}$ Stephan Tenturich war zunächst für den Palatin Ferenc Wesselényi tätig, nach dessen Tod (1666) trat er in den Dienst des Landesrichters Ferenc Nádasdy, unter dem er als „Ambts secretarius” fungierte. ${ }^{29}$ Die Kanzleisekretäre besaßen meistens keinen juristischen Hintergrund, sondern wurden eher aus der Kammerverwaltung rekrutiert und hatten oft bereits in ihren Jugendjahren einige Jahre in der Kanzlei verbracht. Später aber orientierten sie sich häufig in Richtung auf die Obergerichte und schlugen eine juristische Laufbahn ein. Die Ursache dafür liegt in der Organisationsstruktur der Ungarischen Hofkanzlei. Der Posten des Hofkanzlers war bis 1732 Klerikern, später Mitgliedern des Hochadels vorbehalten, so dass die Sekretäre inner-

\footnotetext{
${ }^{27}$ KoltaI, A Lászlóffy-kódex 100.

${ }^{28}$ Miklós Dvornikovich an Adam Batthyány: der gebürtige Rechnitzer Kroate bedankt sich für eine Empfehlung des Grafen, 21. 7. 1653, Ramócz. MNL OL Batthány család levéltára [Familienarchiv Batthyány] P 1314 Missiles Nr. 11057.

${ }^{29}$ Als solcher wird er im ersten Verhör des Grafen Nádasdy am 18. 9. 1670 bezeichnet, ÖStA, HHStA, Ungarische Akten Specialia, Fasc. 313 Konv. C, fol. 38 .
} 
halb der Hofkanzlei über keinerlei Aufstiegsmöglichkeiten verfügten - die Position des Personals bot in dieser Situation eine attraktive Alternative. Es ist also durchaus kein Zufall, dass vier von acht Hofsekretären, die im 17. Jahrhundert dienten, zum Personal aufstiegen: János Lippay (1604-1616), György Orossy (1650-1665), István Orbán (1679-1693) und János Maholányi (1694-1699).30 Um das Bild zu vervollständigen sei erwähnt, dass drei Sekretäre als solche starben (Tiburtius Himmelreich 1610, Lőrinc Ferencffy 1640, András Ruttkay 1650). Ferenc Nagymihály wählte als Einziger einen anderen Karriereweg: Er wurde zum Mitglied des Rates der Ungarischen Hofkammer ernannt (1610). ${ }^{31}$

Auch die Notare bedeuteten eine Verbindung zwischen der Ungarischen Hofkanzlei und dem Gerichtswesen: Einige von ihnen waren nicht nur als Notare der Kanzlei, sondern zugleich als Notare der Königlichen Tafel tätig. Meine Beispiele stammen aus der zweiten Hälfte des 17. Jahrhunderts. In einem Schenkungsbrief aus dem Jahr 1655 wird István Orbán, in einem anderen aus dem Jahr 1659 János Pucz als Notar (Schreiber) der Ungarischen Hofkanzlei und Notar der Königlichen Tafel bezeichnet. ${ }^{32}$ Wie diese Doppelbeschäftigung in der Praxis funktionierte, konnte ich aus dem Archivmaterial nicht klären. Eine andere Verbindung ergab sich im Fall des schon erwähnten Miklós Dvornikovich. Er war neben seiner Hauptbeschäftigung in der Hofkanzlei auch als Beisitzer (assessor) der

\footnotetext{
${ }^{30}$ FALLENBÜCHL, Magyarország főméltóságai 107.

${ }^{31}$ FAZEKAS, Humanisten und Juristen 326-328.

32 Königliche Donation für Mihály Hidvéghy, Gáspár Thalmachy und István Orbán („Cancellariae nostrae Ungaricae et Tabulae Judicariae juratus notarius”) über Güter im Komitat Neutra, 5. 2. 1655, MNL OL MKL A 57 Libri regii vol. 11, pag. 282-283. - Köngliche Donation über drei Dörfer im Komitat Zagreb für János Pucz, 4. 6. 1659, Wien. Ebd. vol. 12, pag. 278-279.
}

Königlichen Tafel tätig. ${ }^{33}$ Problematisch war dies nur in der Zeit der octavarum, in der die Königliche Tafel zusammengerufen wurde und Sitzungen abhielt. Gerade in der Mitte des 17. Jahrhunderts war dies, wie bereits erwähnt, nur sehr unregelmäßig der Fall.

\section{Die Reform der Ungarischen Hofkanzlei 1690 und 1727}

In den letzten Jahrzehnten des 17. Jahrhunderts war vielen ständischen und höfischen Würdenträgern klar, dass die Einrichtungen des ungarischen Königreichs nicht mehr den Anforderungen der Zeit entsprachen und eine Reform dringend notwendig war. In diesen Jahrzehnten entstanden mehrere Reformvorschläge, unter ihnen das berühmte Einrichtungswerk (16881689), das sich auch der Reform der Ungarischen Hofkanzlei und des Gerichtswesens widmete. Eine Trennung beider war noch nicht vorgesehen. ${ }^{34}$ Lediglich eine Neuordnung der Gerichtsbarkeit sah ein Vorschlag aus der Zeit um 1670 vor, in dem auch die Abschaffung der richterlichen Mandate angesprochen wurde. Insbesondere kritisierte der unbekannte Verfasser das Vorgehen der Hofkanzlei auf dem Gebiet der Gerichtsbarkeit: Ihr Einsatz dieses Rechtsmittels führe nicht zur Rechtsbewahrung, sondern eher zu Rechtsunsicherheit:

"Summe igitur et praeprimis necessarium videretur, ipsam cancellariam ad pristinum statum reponi, traductores ejusdem commoneri, ut quisque intra sphaeram sui muneris se contineat, et a turbando antiquo rerum ordine abstineat, neque regiis ex eadem cancellaria expediendis mandatis praeceptoriis,

\footnotetext{
33 Pál Orbán bezeichnet Dvornikovich in der Adresse seines Schreibens folgendermaßen: "SCRM Inclytae Cancellariae Hungaricae registrator et secreti sigilli conservator, tabulae judiciariae regiae juratus assessor", 12. 8. 1661, Galgóc [Hlohovice, Slowakei]. MNL OL MKL A 32 Litterae privatorum Nr. 1008.

${ }^{34}$ KALMÁR, VARGA, Einrichtungswerk 89-104.
} 
contrariis seu inhibitoria, vel dilatoria (siquidem inferiores contra superiorem, praesertim summum judicem, inhibitio nullo jure licita esset) mandata juxta bonum eatenus observari solitum ordinem, et morem extradare praesumat." 35

Die große Reform der Ungarischen Hofkanzlei von 1690 brachte auf diesem Gebiet keine Veränderung. Unter dem erhaltenen Aktenmaterial findet man noch immer in großer Zahl verschiedene Konzepte mit juristischem Inhalt. Die bisherige Praxis veränderte sich erst mit der Neuorganisation des ungarischen Justizwesens auf den Landtagen von 1722/23 und 1729 bzw. mit der neuen Instruktion für die Ungarische Hofkanzlei aus dem Jahre 1727. Die Reformen von 1724 schufen eine ständige Gerichtsorganisation auch auf der mittleren und oberen Ebene: Es wurden vier Distriktualtafeln (tabula judiciaria districtualis) eingesetzt sowie Organisation und Tätigkeit der Königlichen Kurie (Königliche Tafel, Septemviraltafel) neu geregelt. ${ }^{36}$ In dieser Situation wirkten sich die regelmäßigen Eingriffe der Ungarischen Hofkanzlei störend auf die neu formierte Gerichtsbarkeit aus. Deswegen wurden, als die Ungarische Hofkanzlei 1727 eine neue Instruktion bekam (wegen der Einrichtung des Statthaltereirates mussten die Kompetenzen neu aufgeteilt werden), darin auch Bestimmungen aufgenommen, die sich auf ihre Tätigkeit auf dem Gebiet der Gerichtsbarkeit bezogen. ${ }^{37}$ Unter Berufung auf die neue Gerichtsorganisation und die ebenfalls neuen Verfahrensgrundsätze (modus procedendi) wird der Ungarischen Hofkanzlei die Ausstellung verschiedener Mandate untersagt. Die Instruktion erwähnt speziell die mandata inhibitoria, dila-

${ }^{35}$ ÖStA, HHStA, Handschriftensammlung W 185, fol. $447^{r-v}$.

${ }^{36}$ BÓNIS, A bíróság szervezet megújítása; BÓNIS, DEGRÉ, VARGA, A magyar bíróság szervezet 51-53, 6667.

${ }^{37}$ Instruktion der Ungarischen Hofkanzlei, 11. 6. 1727, Wien. MNL OL A 57 Libri regii vol. 35, pag. 595-616, hier 604-605. toria, relaxatoria und cassatoria, die nicht mehr verwendet werden dürfen. Erlaubt sind allein sog. mandata declaratoria, mit denen Informationen über spezielle, außerordentliche Rechtsfälle eingeholt werden konnten. In solchen außerordentlichen Fällen konnte der König auch ein delegiertes Gericht einsetzen.

\section{Die Ungarische Hofkanzlei und die Gerichtsbarkeit nach 1727}

Mit der Instruktion von 1727 hatte die Ungarische Hofkanzlei ihren früheren Einfluss auf die Gerichtsbarkeit verloren. Nur in einigen speziellen Fällen konnte sie noch tätig werden:

I. Der Herrscher übte sein schon erwähntes Gnadenrecht weiterhin durch die Ungarische Hofkanzlei aus. ${ }^{38}$ Noch bis 1848 liefen diese Gnadenakte über die Ungarische Hofkanzlei bzw. nach 1761 auch über den Staatsrat. ${ }^{39}$

II. Die Hofkanzlei beaufsichtigte die Königliche Kurie und konnte, wenn dies für notwendig erachtet wurde, durch ein königliches Reskript in Verfahren eingreifen. Die Bestände der Gerichtsarchive im Ungarischen National- und Staatsarchiv O 10 Normalia, O 11 Rescripta regia (an die Septemviraltafel) und O 12 Rescripta regia (an die Königliche Tafel) beweisen, dass diese Aufsicht wirklich ausgeübt wurde, und zwar bis 1848. Beispielsweise wendeten sich 1818 einige Parteien mit einer Beschwerde gegen das Verfahren der königlichen Tafel an die Ungarische Hofkanzlei. Sie bemängelten, dass die Gerichte ihre Urteile nur kurz begründeten. So könnten die Parteien nicht beurteilen, an welchen Stellen ihre Argumentation fehlerhaft ge-

\footnotetext{
${ }^{38}$ MNL OL MKL A 1 Originales referadae 1727: 52 (Vergiftung des Ehemanns), 1727: 55 (Straßenräuberei), 1728: 16 (Mord).

${ }^{39}$ ÖStA, HHStA, Kabinettsarchiv Staatsrat 1833: 5104 (Mord durch einen Schweinehirten), 1833: 5371 (Vatermord).
} 
wesen sei, wodurch die Vorbereitung der Berufung an die Septemviraltafel erschwert werde..$^{40}$ Wie die anderer Behörden, liefen auch die Personalangelegenheiten der Gerichtshöfe über die Ungarische Hofkanzlei, beginnend mit der Organisation der neuen Rechtsorgane im Jahre 1724.41

III. Noch immer konnte man gegen die Urteile der Königlichen Kurie an den König appellieren (revisio causae); der König blieb nach wie vor summus judex. Es ist leider nicht klar, gegen welche Urteile man sich an den Herrscher wenden konnte. Im Aktenmaterial der Hofkanzlei findet man nur wenige Spuren solcher Fälle. Die bisher nachgewiesenen Beispiele - es handelt sich um solche aus den 1730er Jahren - sind immer spezielle Angelegenheiten. So z.B. der Prozess zwischen den Erben eines Wiener Kaufmanns mit Namen Ferner und dem Freiherrn Johann Gottfried Hellenbach. Letzterer verhandelte in seiner Eigenschaft als Kammergraf der niederungarischen Bergstädte, der vom Kuruzzenfürst Franz Rákóczi II. (1676-1735) ernannt wurde, mit Ferner, und zwar während des Kuruzzenkrieges 1706 und 1707. Wien brauchte ungarisches Rindfleisch, die Aufständischen dagegen Tuch und andere industrielle Produkte. Die Geschäfte liefen offensichtlich mit Genehmigung beider Obrigkeiten. Einige Rechnungen Ferners aber wurden von ungarischer Seite nicht beglichen, so dass dessen Erben diese Forderungen nach zwanzig Jahren (1731-1734) eintreiben wollten. ${ }^{42}$ Die Durchsicht der Akten des österreichischen Staatsrates aus den 1830er Jahren, der

${ }^{40}$ VARGA, Polgári peres eljárás a királyi curián 294295.

${ }^{41}$ MNL OL MKL A 1 Originales referadae 1724: Nr. 15,8 .

${ }^{42}$ MNL OL Familienarchive und Nachlässe P 1568 Nachlass Béla Baranyai Fasz.1. Abschriften aus der sog. "Sammlung Hüttner” (aufbewahrt im Niederösterreichschen Landesarchiv) Bd. 45 (389 ex Nov. 1731, 470 ex Jan. 1732, 488 ex Febr. 1732, 489 ex Febr. 1732, 476 ex Aug. 1734). die als wichtig erachteten Vorträge der Ungarischen Hofkanzlei zu bearbeiten hatte, brachte ein ähnliches Resultat. In diesen Jahren gelangten jährlich etwa 400-450 Stücke aus dem Geschäftsgang der Ungarischen Hofkanzlei auf den Tisch der Staatsräte. Darunter befinden sich immer einige - etwa ein gutes Dutzend -, die mit einem Prozess zu tun hatten. Meistens handelte es sich um Schulden, Erbangelegenheiten sowie Besitzstreitigkeiten bzw. Prozesse zwischen Hochadeligen. ${ }^{43}$

Zusammenfassend kann man festhalten, dass die Ungarische Hofkanzlei dank des ungarischen Rechtssystems des 16. und 17. Jahrhunderts eine nicht zu vernachlässigende Tätigkeit auf dem Gebiet der Gerichtsbarkeit ausübte. Allerdings war ihre Einmischung in das Justizwesen nicht eindeutig positiv, sondern wirkte sich negativ auf das System aus. Nach der Neuorganisation des ungarischen Justizwesens 1724 und der neuen Instruktion für die Hofkanzlei 1727 war diese Tätigkeit nur noch gering und wurde auf spezielle Fälle bzw. auf die Kontrolle der Justizorgane reduziert.

\footnotetext{
${ }^{43}$ ÖStA, HHStA, Kabinettsarchiv Staatsrat 1836: 1675 (Erbangelegenheit zwischen György Gaál von Gyula und seinem Bruder), 1836: 2666 (Schuldenprozess zwischen Anna Podhráczky geb. Spernakovits und Julianna Papanek Miklóssy), 1836: 4121 (Prozess zwischen den Kompossessoren der Gemeinde Csát und der Familie Bene), 1836: 5379 (Prozess zwischen den Familien Esterházy, Vécsey, Bethlen und Bornemissza).
} 


\section{Korrespondenz:}

Dr. István Fazekas

ELTE BTK Középkori és Kora Újkori Magyar

Történeti Tanszék

Múzeum krt. 6-8. I. em. 128, 1088 Budapest

fazekas.istvan@btk.elte.hu

\section{Abkürzungen:}

bes. besonders

Böhm Constantin Edler von Böhm, Die Handschriften des kaiserlichen und königlichen Haus-, Hof- und Staatsarchivs (Wien 1873)

Conc. exp. Conceptus expeditionum

FHKA Finanz- und Hofkammerarchiv

HFU Hoffinanz Ungarn

MKL Magyar Kancellária Levéltára [Archiv der Ungarischen Hofkanzlei]

MNL OL Magyar Nemzeti Levéltár Országos Levéltára [Ungarisches National- und Staatsarchiv, Budapest]

Siehe auch das allgemeine Abkürzungsverzeichnis: [http://www.rechtsgeschichte.at/files/abk.pdf]

\section{Literatur:}

György BóNIS, A bíróság szervezet megújítása III. Károly korában (Systematica commissio) [Die Erneuerung der Organisation der Rechtsprechung in der Zeit Karls III. (Systematica commissio)] (= Értekezések Eckhart Ferenc Jogtörténeti Szemináriumából 5, Budapest 1935).

György BóNIS, Alajos DEGRÉ, Endre VARGA, A magyar bírósági szervezet és perjog története [Die Geschichte des ungarischen Gerichtswesens und Prozessrechtes] (= Levéltári szakmai továbbképzés. Felsőfok 13, Budapest 1961).

Zoltán FALLENBÜCHL, Magyarország főméltóságai [Die höchsten Würdenträger Ungarns] (Budapest 1988).

István FAZEKAS, Die Geschichte der Ungarischen Hofkanzlei (1527-1867) und der Siebenbürgischen Hofkanzlei (1695-1867), in: Gábor UjVÁRY (Hg.), Das ungarische Botschaftsgebäude in Wien. Studien zur Amts- und Kunstgeschichte (Wien 2012) 49-65.

István FAZEKAS, Humanisten und Juristen. Das Personal der Ungarischen Hofkanzlei in der frühen Neuzeit (1526-1690), in: Gábor BÉLI u.a. (Hgg.), Institutions of Legal History with special regard to the legal culture and history (Pécs 2011) 321-331.

János KALMÁR, János J. VARGA (Hgg.), Einrichtungswerk des Königreichs Hungarn (1688-1690) (=Forschungen zur Geschichte und Kultur des östlichen Mitteleuropa 39, Stuttgart 2010).

András KOLTAI, A Lászlóffy-kódex újkori tulajdonosairól [Über die Eigentümer des Codex Lászlóffy in der Neuzeit], in: Magyar Könyvszemle 124 (2008) 93-110.

Martinus Georgius KovACHICH, Formulae solemnes styli in cancellaria, curiaque regum, foris minoribus, ac locis credibilibus, authenticisque Regni Hungariae olim usitati (Pesthini 1799).

Kálmán KovÁcs, Zur Geschichte des ungarischen Strafrechts und Strafprozessrechts 1000-1918: kurzer Überblick (Budapest 1982).

András KUBINYI, Matthias Rex (Budapest 2008).

Theodor MAYER, Verwaltungsreform in Ungarn nach der Türkenzeit (Wien-Leipzig 1911).

Károly MezősI, A fegyverjog (jus armorum) megváltása a töröktől visszafoglalt területeken [Die Ablösung des Waffenrechtes (jus armorum) in den von den Türken zurückeroberten Gebieten], in: Századok 76 (1942) 179-193.

Gottfried MrAZ, Das Palais der Ungarischen Hofkanzlei 1747-1848, in: Lajos GECSÉNYI (Hg.), Das Ungarische Palais in Wien. Die Botschaft der Republik Ungarn (Wien 1994) 37-110.

Anton RADVÁNSZKY, Grundzüge der Verfassungsund Staatsgeschichte Ungarns (=Studia Hungarica 35, München 1990).

Oswald ReDLICH, Weltmacht des Barock. Österreich in der Zeit Kaiser Leopolds I. (Wien ${ }^{41961) . ~}$

István Soós, Pest-Pilis-Solt vármegye és a Neoacquistica Commissio (1696-1713) [Das Komitat Pest-Pilis-Solt und die Neoacquistica Commissio (1696-1713)], in: Ders. (Hg.), Tanulmányok Pest megye monográfiájához [Beiträge zu einer Monographie des Komitats Pest], Bd. 2 (= Előmunkálatok Pest megye monográfiájához 5, Budapest 2008) 17-263.

Ákos TIMON, Ungarische Verfassungs- und Rechtsgeschichte mit Bezug auf die Rechtsentwicklung in den westlichen Staaten (Berlin 1904).

Lajos TORDAY, A megyei polgári peres eljárás a 16.19. században [Das Zivilprozessverfahren in den Komitaten vom 16.-19. Jahrhundert] (Budapest 1933).

Endre VARGA, Polgári peres eljárás a királyi curián 1724-1848/1849 [Das Zivilprozessverfahren an der Königlichen Kurie 1724-1848/1849], in: Levéltári Közlemények 39 (1968) 269-312. 